\title{
Nanoparticle Safety in the Workplace: Developing a Methodology to Monitor and Remediate Spills.
}

\author{
Stefano Rubino ${ }^{1}$, Brad Lute ${ }^{1}$, Iris Guo ${ }^{1}$, Kelly Cadieux ${ }^{1}$, Byron D. Gates ${ }^{1}$. \\ ${ }^{1 .}$ Department of Chemistry and 4D LABS, Simon Fraser University, Burnaby, BC, Canada.
}

Recent years have seen a rapidly increasing presence of nanoparticles not only in research applications, but also in industries such as medical and manufacturing. Since nanoparticles often exhibit properties unlike those of their bulk counterparts, they should be treated as unknown substances with potentially dangerous collateral effects until long-term assessments are completed. The field of nanotoxicity has recently received much attention from governments and public agencies, with the establishment of national and international infrastructures targeted at studies on safe use of nanomaterials (for example, the EU funded QualityNano research infrastructure).

We propose here a methodology to monitor and remediate spills of nanoparticles in the workplace, with the aim to develop a policy on safe usage and handling of nanomaterials in general. Ideally, the methods developed should be easy to apply, inexpensive and non-destructive to the workplace.

Nanoparticle spills are often invisible to the naked eye, but several techniques can be used to detect them on a work surface. In this study, we considered a common laboratory countertop contaminated by spills of solutions containing nanoparticles in different solvents. A complete characterization of the spill would require tools with elemental resolution in the nanometer scale. However, we assume that the contaminant nanoparticle characteristics are already well known to a given workplace, and instead focus on features that allow us to easily distinguish them from the countertop itself or common macroscale contaminants. For example, such features of contaminant nanoparticles could be element(s) not present in the workplace, specific optical properties or a unique shape.

We have chosen as representative examples Au nanorods, Se nanowires and FePt nanoparticles. We selected X-Ray Fluorescence (XRF) as an optimal tool to detect spills based on previous results obtained in our research group [1,2]. The detection limits for XRF are established by direct comparison with other investigative analytical tools, such as Inductively Coupled Plasma Mass Spectrometry (ICP-MS), light absorption spectroscopy (Fig. 1), and Scanning and Transmission Electron Microscopy (SEM/TEM) (Fig. 2) coupled with Energy Dispersive X-ray Spectroscopy (EDXS).

Once a spill is detected, various remediation strategies can be applied. It was shown that wiping of the spill is ineffective, but also spreads the nanoparticles over a larger area [1,2]. More effective strategies involve the casting of a polymer or rubber on the spill to encapsulate the nanoparticles. Depending on the particles, remediating agent(s), and surface interactions, a varying number of applications of the remediating agent are necessary to remove a significant number of nanoparticles from the contaminated surfaces. The efficacy of the remediation is monitored using the same techniques mentioned above.

The method is tested for a wide number of parameters to ensure general applicability in conditions commonly encountered in the workplace. Such parameters include: i) nanoparticle composition, shape and their surface chemistry; ii) solvent composition and polarity; iii) remediation agents; and iv) use of surfactants or other additives prior to applying the remediation techniques. Spills containing more than 
one type of nanoparticle are investigated as well, to determine the effect of particle interaction on detection and remediation.

\section{References:}

[1] J Zhou, M.Sc. Thesis, Simon Fraser University (2015).

[2] BD Gates et al, "Detecting, Handling and Controlling Nanoparticle Contamination in the Workplace, Final Report for WorkSafeBC and Workers’ Compensation Board of Nova Scotia”, Project number: RS2010-IG40, (2013).

[3] We thank Brandy K. Pilapil and Michael Wang for providing FePt and Se nanomaterials used in this work. This research was supported in part by funds from WorkSafeBC through the Innovation at Work program, the Natural Sciences and Engineering Research Council (NSERC) of Canada, the Canada Research Chairs Program (B.D. Gates), and CMC Microsystems through the MNT Financial Assistance program that facilitated access to materials characterization services. This work made use of 4D LABS shared facilities supported by the Canada Foundation for Innovation (CFI), British Columbia Knowledge Development Fund (BCKDF), Western Economic Diversification Canada, and Simon Fraser University.
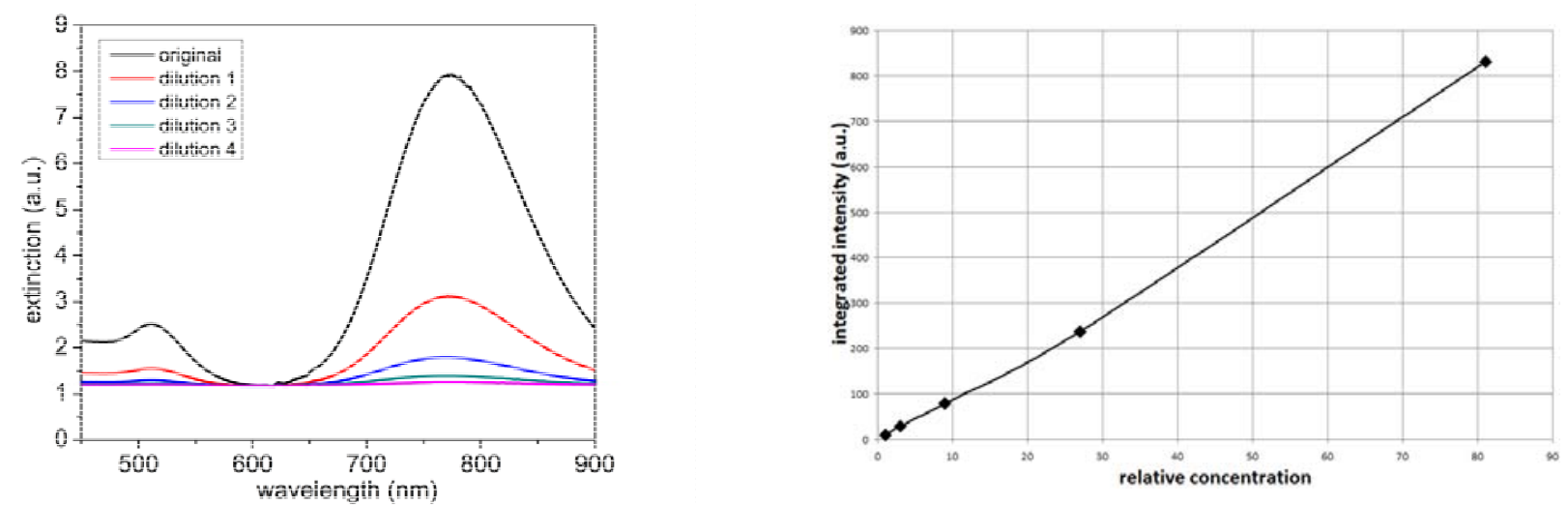

Figure 1. Left) Light absorption spectra of the Au nanoparticle solutions prepared by serial dilution, showing the plasmonic peaks characteristic of Au nanorods. Right) Plot of the integrated intensity of the absorption spectra in the region [680-860] $\mathrm{nm}$ around the peak centered at $780 \mathrm{~nm}$ as function of the relative nanoparticle concentration.
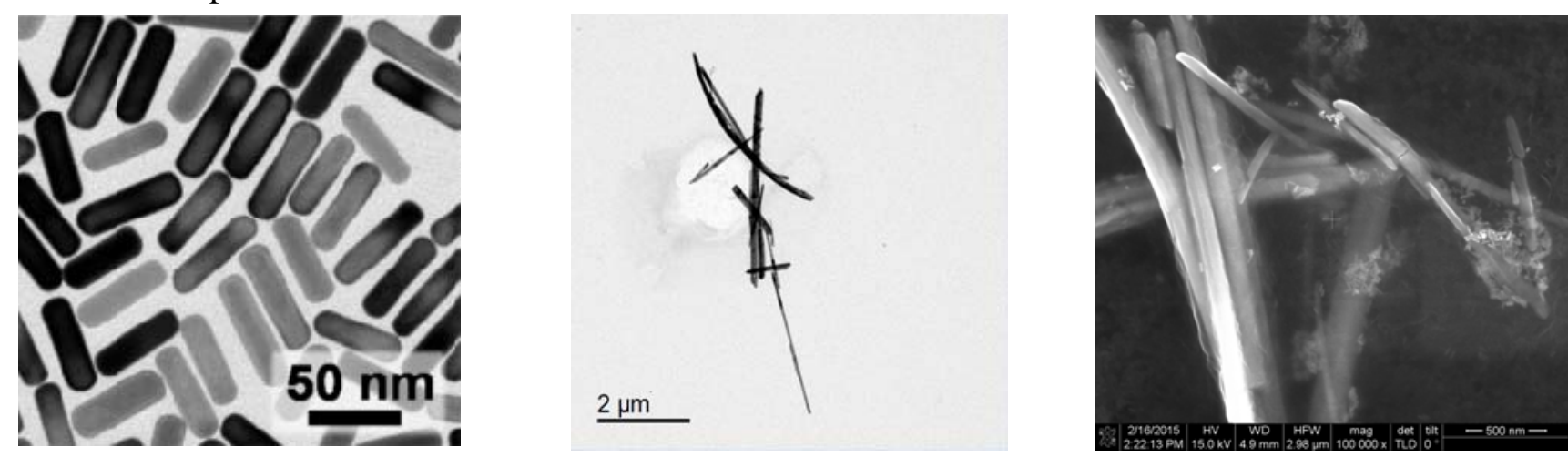

Figure 2. TEM micrographs of Au nanorods (left) and Se nanowires (middle) dropcast on a TEM grid. SEM image of a simulated spill containing both types of nanomaterials (right). The shape and aspect ratio of these nanoparticles makes them easily distinguishable from other sources of contamination. 